\title{
Comportamentos de risco à saúde: uma análise com estudantes da rede pública de ensino
}

\author{
Health risk behaviors: an analysis with \\ students of the public school system
}

\author{
Raiane da Rosa Dutra ${ }^{1}$, Renato Xavier Coutinho ${ }^{2}$
}

\begin{abstract}
A aptidão física contribui para o desenvolvimento de atividades do cotidiano onde a prática de exercício físico possui importância significativa na incorporação de um estilo de vida saudável. Neste contexto, o presente trabalho teve como objetivo analisar medidas antropométricas e aptidão física de jovens estudantes de escolas públicas, com o intuito de verificar comportamentos de risco entre os mesmos. O procedimento metodológico do estudo foi comparativo descritivo; verificou-se massa corporal e estatura, além da realização de testes de aptidão física relacionados à saúde de 231 estudantes, que foram divididos por sexo e faixa etária para análise. Observou-se que as médias, em sua maioria, encontram-se dentro do esperado como "desejável" para cada faixa etária, porém, destacaram-se os níveis máximos e mínimos das variáveis, indicando possíveis exposições a comportamentos de risco relacionados à saúde. Constatou-se que os meninos possuem maior aptidão física referente aos testes de força muscular local e resistência cardiorrespiratória, e os adolescentes de 14 a 17 anos obtiveram melhores resultados no teste de força muscular local. Com isso, se faz importante construir propostas de intervenções pedagógicas centradas no ensino pela pesquisa, promovendo discussão e reflexão quanto aos hábitos de vida dos jovens.
\end{abstract}

Palavras-chave: Aptidão física. Jovens. Escola.

\footnotetext{
${ }^{1}$ Doutoranda em Educação em Ciências: Química da Vida e Saúde na Universidade Federal de Santa Maria (UFSM), Santa Maria, Rio Grande do Sul, Brasil. E-mail: raiane.rosa.dutra@gmail.com

${ }^{2}$ Doutorado em Educação em Ciências: Química da Vida e Saúde pela Universidade Federal de Santa Maria, Santa Maria, Rio Grande do Sul, Brasil. Professor do Instituto Federal Farroupilha (IFFar), campus São Vicente do Sul, São Vicente do Sul, Rio Grande do Sul, Brasil. E-mail: renato.coutinho@iffarroupilha.edu.br
} 


\begin{abstract}
Physical fitness contributes to the development of daily activities where the practice of physical exercise has significant importance in incorporating a healthy lifestyle. In this context, the present study aimed to analyze anthropometric measures and physical fitness of young students of public schools, in order to verify risk behaviors among them. The methodological procedure of the study was descriptive comparative; Body mass, stature, and health-related physical fitness tests were performed for 231 students, who were divided by sex and age group for analysis. It was observed that the averages, for the most part, are within what was expected to be "desirable" for each age group, but the maximum and minimum levels of the variables were highlighted, indicating possible exposures to health-related risk behaviors. It was verified that the boys had greater physical fitness regarding the tests of local muscular strength and cardiorespiratory resistance, and the adolescents of 14 to 17 years obtained better results in the test of local muscle strength. Thus, it is important to build proposals for pedagogical interventions focused on teaching through research, promoting discussion and reflection on the life habits of young people.
\end{abstract}

Keywords: Physical fitness. Young. School.

\section{Introdução}

“Uma boa aptidão física é caracterizada pela comunicação harmônica entre indicadores de saúde e desempenho físico, onde os componentes de saúde garantem a homeostase corporal e o desempenho físico, que contribuem para as atividades do cotidiano. A aptidão física atrelada à saúde associa-se aos componentes: capacidade cardiorrespiratória, níveis de composição corporal, flexibilidade e resistência muscular." ${ }^{(1-2)}$

Goersch et al. ${ }^{(3)}$ assinalam que a prática de exercício físico, voltado para a aptidão física relacionada à saúde, possui importância significativa na incorporação de um estilo de vida fisicamente ativo. Conforme Pesquisa Nacional de Saúde, realizada pelo Instituto Brasileiro de Geografia e Estatística - IBGE, ${ }^{(4)}$ o estado do Rio Grande do Sul apresenta o maior percentual de pessoas com excesso de peso, cerca de $63 \%$ da sua população, enquanto a média nacional é de $56,9 \%$, sendo mais comum entre as mulheres gaúchas.

Por essa razão, o ambiente escolar tem papel importante na promoção de atividade física entre os jovens. Cardoso et al. ${ }^{(5)}$ apontam que "sob o prisma pedagógico a Educação Física Escolar (EFE) é uma alternativa de intervenção em contrapartida aos preocupantes dados de crianças e adolescentes, com baixos níveis de aptidão física relacionada à saúde e estilo de vida sedentário."

Quanto às competências específicas de Educação Física para o Ensino Fundamental, a Base Nacional Comum Curricular - BNCC expõe que "é possível assegurar aos alunos a (re) construção de um conjunto de conhecimentos que permitam ampliar sua consciência a respeito de seus movimentos e dos recursos para o cuidado de si e dos outros e desenvolver autonomia para apropriação e utilização da cultura corporal de movimento em diversas finalidades humanas, favorecendo sua participação de forma confiante e autoral na sociedade."(6)

Quanto às Diretrizes Curriculares Nacionais para o Ensino Médio - DCN's, a Educação Física escolar tem papel fundamental para que os jovens consigam compreender os saberes vinculados às práticas corporais, visto que "[...] raramente se têm disseminadas compreensões mais abrangentes que nos permitam entender que o crescimento intelectuale afetivo não se realizam sem um corpo, e que, enquanto uma das dimensões do humano, tem sua concepção demarcada histórico-culturalmente. Desse modo, ao educador é imprescindível tomar o educando nas suas múltiplas dimensões - intelectual, social, física e emocional - e situálas no âmbito do contexto sócio-cultural em que educador e educando estão inseridos."(7) 
Dessa forma, a escola tem papel fundamental na construção de uma consciência crítica e reflexiva em relação à representatividade das práticas corporais, com a função de colaborar para a transformação dos sujeitos expostos a comportamentos sedentários.

Neste contexto, o presente trabalho teve como objetivo analisar medidas antropométricas e aptidão física de jovens escolares, com o intuito de verificar comportamentos de risco referentes à aptidão física relacionada à saúde.

\section{Material e Método}

O procedimento metodológico foi comparativo descritivo, conforme Thomas e Nelson, ${ }^{(8)}$ no qual, adaptado de Coutinho et al. (2014), ${ }^{(9)}$ realizou-se a verificação da massa corporal e percentual de gordura, estatura e testes de aptidão física relacionados à saúde, conforme protocolo do Projeto Esporte Brasil - PROESP, ${ }^{(10)}$ sendo estes: teste de flexibilidade (sentar e alcançar com banco de Wells), teste de força muscular local/resistência abdominal e teste de resistência cardiorrespiratória (6 minutos) durante as aulas de educação física. E foram utilizados banco de Wells (Sanny), balança (modelo digital de alta precisão, Millenium, capacidade de 150 quilogramas e precisão de 100 em 100 gramas), cronômetro digital (Kikos, Cr 60 - 60 voltas), estadiômetro (Filizola), prancheta, caneta, ficha de avaliação, colchonetes e cones.

A pesquisa foi desenvolvida com jovens escolares de quatro escolas (duas estaduais e duas municipais) de um município localizado na Região Central do estado do Rio Grande do Sul. Consideramos importante descrever algumas características dos espaços de práticas de atividades físicas das escolas e da comunidade, para que seja possível vislumbrar o contexto em que os sujeitos participantes estão inseridos:

- Todas as escolas possuem quadras cobertas, no entanto, apenas três delas ficam à disposição dos estudantes para a prática de atividades fora do período escolar;
- Na praça municipal há uma quadra de esportes aberta e academia ao ar livre; um campo de futebol com pista de caminhada/corrida no entorno e ciclovia num trecho da cidade.

Foram entregues termos de Assentimento e Consentimento Livre e Esclarecido para 611 estudantes que foram informados sobre a preservação do anonimato, garantindo-lhes que os dados seriam utilizados apenas na pesquisa. Como critério de exclusão utilizou-se a não entrega dos termos e/ou a não autorização dos pais para participar da pesquisa. A amostra final foi composta por 231 estudantes, com faixa etária entre 11 e 17 anos, sendo $124(53,7 \%)$ do sexo feminino e 107 $(46,3 \%)$ do sexo masculino.

Os dados coletados foram organizados em um banco de dados desenvolvido no Excel $^{\circledR}$, onde foram calculados valores médios, máximos e mínimos de cada variável; e analisados no programa SPSS Statistics 17.0, utilizando estatística descritiva, com o teste para amostras independentes de Mann-Whitney (Teste U) com as variáveis: sexo, idade, flexibilidade, força muscular local e resistência (6min), onde os dados foram considerados significativamente diferentes quando o $p<0,05$. Os estudantes foram divididos por sexo (feminino e masculino) e por faixa etária: Grupo 1 - de 11 a 13 anos; e Grupo 2 - de 14 a 17 anos.

Esta pesquisa foi aprovada pelo Comitê de Ética e Pesquisa sob Número do Parecer: 2.310.003.

\section{Resultados}

Analisando as médias do sexo feminino, observou-se que as médias do percentual de gordura foram maiores no grupo 2 - de 14 a 17 anos, assim como de IMC, teste de flexibilidade e força muscular local; já a média da distância percorrida no teste de corrida $(6 \mathrm{~min})$ mostrou-se maior no grupo 1 - de 11 a 13 anos. No sexo masculino, notou-se que a média de percentual de gordura foi maior no grupo 1 - de 11 a 13 anos; e a média de IMC, teste de flexibilidade, força muscular local e corrida (6min) encontraram-se maiores no grupo 2 - de 14 a 17 anos, conforme Tabela 1. 
Tabela 1 - Análise de médias, máximos e mínimos das variáveis conforme sexo e faixa etária.

\begin{tabular}{|c|c|c|c|c|c|c|c|}
\hline \multirow{3}{*}{ Faixa etária } & \multirow{3}{*}{$\begin{array}{l}\text { Variáveis } \\
\text { analisadas }\end{array}$} & \multicolumn{6}{|c|}{ Sexo } \\
\hline & & \multicolumn{3}{|c|}{ Feminino } & \multicolumn{3}{|c|}{ Masculino } \\
\hline & & Média & Máximo & Mínimo & Média & Máximo & Mínimo \\
\hline 11 a 13 anos & $* \%$ de gordura & 23,9 & 57,3 & 10,8 & 16,6 & 37,4 & 4 \\
\hline \multirow{2}{*}{ Meninas $(\mathrm{N}=64)$} & *IMC & 21,1 & 30,6 & 15,5 & 21,1 & 30 & 15,2 \\
\hline & Flexibilidade & 24,7 & 43 & 9 & 23,3 & 40 & 11 \\
\hline \multirow[t]{2}{*}{ Meninos (N=44) } & *Força M. L. & 25,2 & 49 & 2 & 31,8 & 50 & 15 \\
\hline & Corrida (6min) & 1121 & 1874,4 & 630,6 & 1322,3 & 2368,8 & 798 \\
\hline 14 a 17 anos & $* \%$ de gordura & 25,2 & 52,2 & 13,5 & 16,5 & 40,1 & 6,3 \\
\hline \multirow[b]{2}{*}{ Meninas $(\mathrm{N}=60)$} & *IMC & 22,3 & 44,7 & 16,7 & 21,4 & 39,5 & 14,2 \\
\hline & Flexibilidade & 25,9 & 37 & 14 & 25,9 & 39 & 11 \\
\hline \multirow[t]{2}{*}{ Meninos $(\mathrm{N}=63)$} & *Força M. L. & 27,6 & 60 & 7 & 42 & 67 & 21 \\
\hline & Corrida (6min) & 1028,5 & 1760,8 & 564,3 & 1438,9 & 2260,5 & 558 \\
\hline
\end{tabular}

Fonte: Autores

*\% de gordura: percentual de gordura. *IMC: Índice de Massa Corporal. *Força M. L.: força muscular local.

$\mathrm{Na}$ análise para amostras independentes $(\mathrm{p}=0,000)$ e resistência cardiorrespiratória - 6min observou-se associação significativa entre a ( $=0,000)$; e a variável "idade" com o teste de força variável "sexo" e os testes de força muscular local muscular local $(\mathrm{p}=0,000)$, conforme a Tabela 2.

Tabela 2 - Análise para amostras independentes.

\begin{tabular}{rcccc}
\hline \multicolumn{1}{l}{ Variáveis } & $\mathbf{N}$ & Flexibilidade & Força M. L. & Resistência (6min) \\
\cline { 3 - 5 } & & $\mathbf{P}$ & $\mathbf{P}$ & $\mathbf{P}$ \\
\hline Sexo - Masculino & 107 & 0,437 & 0,000 & 0,000 \\
Feminino & 124 & & & \\
Idade - De 11 a 13 & 108 & 0,099 & 0,000 & 0,877 \\
De 14 a 17 & 123 & & & \\
\hline
\end{tabular}

Fonte: Autores

\section{Discussão}

A média de percentual de gordura encontrada entre o sexo feminino no grupo 2 foi de 25,2, sendo maior que a encontrada no grupo 1, de 23,9. Conforme Lohman, ${ }^{(1)}$ o percentual de gordura indicado como na "média" entre o sexo feminino é de 23, configurando assim que os valores encontram-se "acima da média". O mesmo foi observado para os grupos do sexo masculino com médias de 16,6 e 16,5 respectivamente, sendo ainda, segundo Lohman, ${ }^{(11)}$ o valor ideal de 15.

Conforme Momm e Höfelmann, (12) "fatores que podem causar o excesso de peso são: aumento 
do consumo de alimentos com alto teor de lipídio, sacarose e sódio; redução do consumo de cereais integrais, frutas e hortaliças; inatividade física devido ao uso de computadores, jogos eletrônicos e televisores." Silva et al. ${ }^{(13)}$ apontam que na adolescência a atividade física reduz o percentual de gordura corporal, além de proporcionar benefícios à saúde óssea e contribuir para a melhora do perfil lipídico e metabólico.

A média de IMC observada no grupo 1 e 2 do sexo feminino foi de 21,1 e 22,3 respectivamente; segundo indicadores do PROESP-BRASIL, ${ }^{(14)}$ configuram-se como parâmetros de normalidade, sendo a média indicada para estes grupos de 22,3 e 23,1, nesta ordem. Quanto ao sexo masculino, os grupos 1 e 2 encontram-se dentro do padrão de normalidade $(21,1 ; 21,4$, nesta ordem) sendo o indicado 22,1 e 23,7 respectivamente. Conforme Guedes et al., ${ }^{(15)}$ identificar possíveis inadequações nutricionais em segmentos específicos da população jovem podem oferecer subsídios para programas de intervenção em Saúde Pública.

Capel et al. ${ }^{(16)}$ colocam que estudos realizados em escolas públicas e privadas no Brasil demonstram resultados preocupantes, alertando para os perigosos efeitos advindos da falta de exercício físico rotineiro e aumento do sedentarismo nas crianças e adolescentes. A verificação do IMC e percentual de gordura se constituem como indicadores de saúde, e segundo Caltran et al.(17) "o IMC de populações gera indicadores para identificar grupos que necessitam de intervenção nutricional. Para o campo da saúde pública esta avaliação se mostra uma ferramenta de grande utilidade. O cálculo do IMC é um dos métodos mais simples, considerado de fácil aplicação e baixo custo."

No entanto, ainda conforme Caltran et al., ${ }^{(17)}$ quando comparado a outros métodos de avaliação da composição corporal, o IMC demonstra uma estimativa imprecisa da massa de gordura e massa magra, pois não fornece informação sobre alterações de peso. Dessa forma, o IMC não reflete a real composição corporal.

No teste de flexibilidade entre o sexo feminino, observou-se níveis de desempenho acima da média nos grupos 1 e $2(24,7 \mathrm{~cm} ; 25,9$ $\mathrm{cm}$ respectivamente), sendo o indicado, conforme o PROESP-BRASIL, $23,5 \mathrm{~cm}$ para meninas de 11 a 13 anos, e 24,3 cm para meninas de 14 a 17 anos. Entre o sexo masculino se observou no grupo 1 média abaixo do indicado $(23,3 \mathrm{~cm})$, sendo o desejável 25,2 cm, indicando risco à ocorrência de desvios posturais e queixa de dores nas costas. $\mathrm{O}$ grupo 2 apresentou média dentro dos padrões de normalidade $(25,9 \mathrm{~cm})$ sendo o indicado $24,7 \mathrm{~cm}$. Nogueira e Pereira, ${ }^{(18)}$ ao analisarem a prevalência de atendimento aos critérios de Atividade Física Relacionada à Saúde e associação com o sexo, a faixa etária e o tempo de participação num programa social esportivo em adolescentes de Fortaleza, Ceará (CE), mostraram que, em comparação com as meninas, os meninos apresentaram maior dificuldade em atender os critérios de flexibilidade.

No teste de força muscular local (abdominal), foi observado entre o sexo feminino médias de 25,2 para o grupo 1 e de 27,6 para o grupo 2, encontrando-se dentro dos parâmetros desejados segundo o PROESP-BRASIL, de 21 e 23 na devida ordem. Entre o grupo masculino foram encontradas médias também desejáveis em ambos os grupos (31,8 e 42 respectivamente), consistindo como níveis indicados para a faixa etária do grupo 1, 30 abdominais, e para a faixa etária do grupo 2, 39 abdominais. Gadelha et al ${ }^{(19)}$ colocam que, com a prática regular de exercícios há o aumento da força muscular, relevante para a redução de riscos de lesões nas atividades diárias, além da possível melhora nos escores da qualidade de vida.

Para o teste de resistência cardiorrespiratória (corrida 6min), verificou-se entre o sexo feminino médias de $1121 \mathrm{~m}$ para o grupo 1, encontrandose segundo os parâmetros do PROESP-BRASIL dentro do indicado para esta faixa etária $(963 \mathrm{~m}) \mathrm{e}$ $1028,5 \mathrm{~m}$ para o grupo 2, configurando-se abaixo do indicado $(1125 \mathrm{~m})$, sendo um indicador de risco à presença de níveis elevados de colesterol e pressão arterial, além da provável ocorrência de obesidade. No grupo do sexo masculino, tanto o grupo 1, quanto o grupo 2 obtiveram médias satisfatórias $(1322,3 \mathrm{~m}$ e $1438,9 \mathrm{~m}$ 
respectivamente), sendo o indicado médias de 1000 m e 1159 m, nesta ordem. Sehn et al., ${ }^{(20)}$ ao verificarem os níveis de aptidão física relacionada à saúde em escolares, observaram que no teste de aptidão cardiorrespiratória ocorreram baixos níveis no sexo feminino.

As médias das variáveis analisadas no presente estudo, de maneira geral, encontraram-se dentro dos índices indicados segundo os parâmetros do PROESP-BRASIL. ${ }^{(14)}$ No entanto, é importante destacar que os valores mínimos de flexibilidade, resistência muscular local e resistência cardiorrespiratória (6min) apontam para possíveis casos de sedentarismo e, consequentemente, exposição ao desenvolvimento de DCNT.

Analisando a associação significativa entre a variável "sexo" e o teste de força muscular local, observou-se que os meninos possuem maior resistência muscular quando comparados às meninas, assim como resistência cardiorrespiratória (corrida 6min). Ribeiro et al., ${ }^{(21)}$ ao avaliarem a aptidão física relacionada à saúde de homens e mulheres, apontam que diferenças significativas foram encontradas nos testes de resistência cardiorrespiratória e força muscular local, nos quais a proporção de homens que atenderam aos critérios foi maior em relação às mulheres.

Distinto ao que foi observado na presente análise, Contreira et al., ${ }^{(22)}$ ao investigarem o perfil de aptidão física relacionada à saúde de adolescentes de Florianópolis (SC), segundo as referências da bateria PROESP/BR, constataram que a maioria dos escolares apresentou desempenho na zona de risco para os resultados referentes à força/resistência abdominal, com tendência para os meninos nesta classificação; em relação aos testes de função cardiorrespiratória, todos os escolares avaliados foram classificados na zona de risco, o que os autores consideram preocupante para as questões relativas à saúde.

Ainda, Contreira et al..$^{(22)}$ apontam que "observando tais aspectos, reflete-se sobre a importância da atuação dos professores de educação física, no sentido de estimular os alunos durante as aulas de educação física para a manutenção das práticas de atividades física e hábitos alimentares saudáveis, a fim de que esses hábitos sejam agregados em outras fases da vida".

Quanto à associação significativa entre a variável "idade" e força muscular local, foi possível observar que o grupo 2 obteve melhores resultados em comparação com o grupo 1. É correto afirmar que a resistência muscular está ligada diretamente com os níveis de atividade física desempenhada pelos indivíduos onde, num estudo desenvolvido por Santos et al., ${ }^{(23)}$ os autores verificaram que em relação aos índices de atividade física comparados por faixa etária, em ambos os sexos, quanto maior a faixa etária menores foram os índices de atividade física apresentados pelos adolescentes, diferenciando-se, assim, do resultado encontrado no presente estudo.

Diante dos altos índices de sobrepeso e obesidade, a OMS lançou novas diretrizes de combate à obesidade infantil no mundo, pois estima-se que 41 milhões de crianças menores de cinco anos sejam obesas ou estejam acima do peso. Essas incluem aconselhamento e dieta, avaliação dos hábitos alimentares, além das mais comuns medições de peso e altura. ${ }^{(24)}$ Tais prevalências de sobrepeso e obesidade refletem mudanças comportamentais que privilegiam dietas não saudáveis e inatividade física.

\section{Conclusão}

Observou-se que as médias de IMC, flexibilidade em ambos os grupos do sexo feminino e no grupo 2 do sexo masculino, força muscular local e teste de resistência cardiorrespiratória para o grupo 1 do sexo feminino e ambos os grupos do sexo masculino, encontram-se dentro do esperado como "desejável"; porém, chamaram a atenção os níveis máximos e mínimos das variáveis analisadas, indicando possíveis exposições a comportamentos de risco relacionados à saúde. Ainda, quanto às associações significativas analisadas, constatouse que os meninos possuem maior aptidão física referente aos testes de força muscular local e resistência cardiorrespiratória, porém, estudos 
apontam que, quando comparados às meninas, os meninos são fisicamente mais ativos. Além disso, houve associação significativa quanto à faixa etária e força muscular local, onde os jovens de 14 a 17 anos obtiveram melhores resultados, o que pode ser explicado devido às mudanças hormonais $\mathrm{e}$ fisiológicas características desta fase.

Com isso, sugerem-se propostas de intervenção pedagógica que tenham como objetivo o ensino pela pesquisa, onde através de dados da comunidade escolar os alunos possam ser atuantes na construção do conhecimento, e que tenham também como objetivo a mudança de índices inadequados; estes objetivos apresentam-se como uma maneira de significar a importância dos alunos no processo de ensino e aprendizagem.

\section{Agradecimentos}

À Coordenação de Aperfeiçoamento de Pessoal de Nível Superior (Capes), pelo apoio financeiro.

\section{Referências}

1 Andreasi V, Michelin E, Rinaldi AEM, Burini RC. Physical fitness and associations with anthropometric measurements in 7 to 15 -year-old school children. J Pediatr. 2010;86(6):497-502.

2 Mello JB, Hernandez MS, Farias VM, Pinheiro ES, Bergmann GG. Aptidão física relacionada ao desempenho motor de adolescentes de Uruguaiana, Rio Grande do Sul. Rev Bras Ciênc Mov. 2015;23(4):72-9.

3 Goersch CM, Lamboglia F, Costa RG, Franchi KMB, Pequeno LL, Pinheiro MHNP. Efeito do tempo de prática de exercício físico na aptidão física relacionada à saúde em mulheres idosas. Rev Bras Promoç Saúde. 2014;27(1):29-36.

4 Instituto Brasileiro de Geografia e Estátistica. Pesquisa Nacional de Saúde 2015 [Internet]. 2015 [citado 2018 maio 23]. Disponível em: ftp://ftp.ibge.gov.br/PNS/2015/pns2015.pdf
5 Cardoso MA, Pereira FM, Afonso MR, Rocha Junior IC. Educação física no ensino médio: desenvolvimento de conceitos e da aptidão física relacionados à saúde. Rev Bras Educ Fís Esporte. 2014; 28(1): 147-61.

6 Ministério da Educação (BR). Base Nacional Comum Curricular: ensino fundamental. Brasília: MEC; 2017.

7 Ministério da Educação (BR). Diretrizes Curriculares Nacionais: ensino médio. Brasília: MEC; 2013.

8 Thomas JR, Nelson JK. Métodos de pesquisa em atividade física. 3a. ed. Porto Alegre: Artmed; 2002.

9 Coutinho RX, Donadel NJ, Santos WM, Carijó DN, Noro SJ. Avaliação da aptidão física como estratégia de contextualização das aulas de Educação Física no ensino médio. EFDeportes [Internet]. 2014 [citado 2019 mar 4];18(188). Disponível em: https://www.efdeportes.com/ efd 188/avaliacao-da-aptidao-f\%C3\%ADsicano-ensino-medio.htm

10 Projeto Esporte Brasil. Bateria de testes [Internet]. 2017 [citado 2017 mar 15]. Disponível em: https://www.ufrgs.br/proesp/ bateria-de-testes.php

11 Lohman TG. Advances in body composition assessment: current issues in exercise science series. Champaign: Human Kinetics; 1992.

12 Momm N, Höfelmann DA. Qualidade da dieta e fatores associados em crianças matriculadas em uma escola municipal de Itajaí, Santa Catarina. Cad Saúde Colet. 2014;22(1):32-9.

13 Silva MC, Engers PB, Vilela GF, Spohr CF, Rombaldi AJ. Fontes de informação sobre benefícios à prática de atividade física e fatores associados em adolescentes: estudo de base escolar. Rev Bras Ativ Fis Saúde. 2016;21(3):237-45.

14 Projeto Esporte Brasil. Avaliação da aptidão física relacionada à Saúde (APFS) [Internet]. 2018 [citado 2018 abr 27]. Disponível em: https://www.ufrgs.br/proesp/nc-apfs.php 
15 Guedes DP, Almeida FN, Miranda Neto JT, Maia MFM, Tolentino TM. Baixo peso corporal/magreza, sobrepeso e obesidade de crianças e adolescentes de uma região brasileira de baixo desenvolvimento econômico. Rev Paul Pediatr. 2013;31(4):437-43.

16 Capel TL, Vaisberg M, Araújo MP, Paiva RFL, Santos JMB, Bella ZIKJ. Influência do índice de massa corpórea, porcentagem de gordura corporal e idade da menarca sobre a capacidade aeróbia (VO2 máx) de alunas do ensino fundamental. Rev Bras Ginicol Obstet. 2014;36(2):84-9.

17 Caltran P, Silva SC, Pope S, Fornari JV, Barnabé AS, Arçari DP, et al. Utilização do índice de massa corporal para estimativa do estado nutricional de funcionários de uma empresa do ramo químico. Educ Foco. 2013;5(6):103-12.

18 Nogueira JAD, Pereira CH. Aptidão física relacionada à saúde de adolescentes participantes de programa esportivo. Rev Bras Educ Fís Esporte. 2014;28(1):31-40.

19 Gadelha VB, Sardeli AV, Santos WM, Ribeiro $\mathrm{CP}$, Moraes DFG, Cavaglieri CR, et al. Importância da força muscular para a qualidade de vida de idosos sedentários. Rev Bras Qual Vida. 2017;9(2):153-64.

20 SehnAP, Reuter CP, Kern DG, Silva CF, Barbian $\mathrm{CD}$, Welser L, et al. Perfil sociodemográfico associado em nível de Aptidão Física Relacionada à Saúde em escolares. Rev Saúde Pesqui. 2017; 10(1): 75-82.

21 Ribeiro AS, Silva DRP, Carvalho FO, Schiavoni D, Jesus BCS, Cyrino ES. Aptidão física relacionada à saúde em homens e mulheres de 17-26 anos. Rev Bras Ativ Fis Saúde. 2013;18(2):197-204.

22 Contreira AR, Pizzo GC, Rocha FF, LazierLeão TR, Caruzzo AM, Copetti F, et al. Perfil de aptidão física relacionada à saúde em adolescentes. Rev Saúde Pesqui. 2016; 9(2): 309-15.
23 Santos GC, Stabelini Neto A, Sena JS, Campos W. Atividade física em adolescentes: uma comparação entre os sexos faixas etárias e classes econômicas. Rev Bras Ativ Fis Saúde. 2014;19(4):455-64.

24 Organização das Nações Unidas. OMS lança novas diretrizes de combate à obesidade infantil no mundo [Internet]. 2017 [citado 2018 maio 14]. Disponível em: https://nacoesunidas. org/oms-lanca-novas-diretrizes-de-combate-aobesidade-infantil-no-mundo/ 